\title{
Gel 1
}

Marker and peak fraction F3 are shown in the lower panel of Fig 1A.

Non-included lanes are marked with an ' $\mathrm{X}$ '.

Full gel is shown in S1A Fig.

Uncropped Coomassie stained SDS-PAGE gel of fractions (F1 - F7) collected during size-exclusion chromatography of affinity purified Fl-C9orf72 - Fl-SMCR8 - Fl-WDR41 complex.

Abbreviations: M, marker; MW, molecular weight; F, fraction; V, void

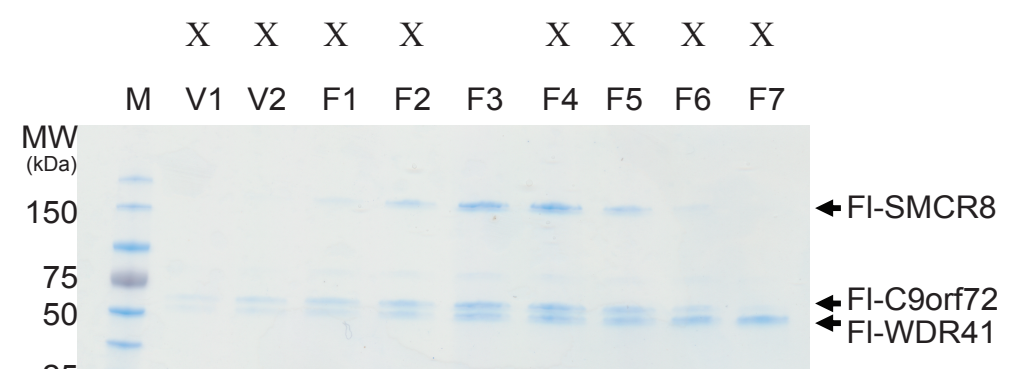




\section{Gel 2}

Marker and peak fraction F4 are shown in the lower panel of Fig 1B.

Non-included lanes are marked with an ' $\mathrm{X}$ '.

Full gel is shown in S1C Fig.

Uncropped Coomassie stained SDS-PAGE gel of fractions (F1 - F6) collected during size-exclusion chromatography of affinity purified Fl-C9orf72 - SMCR $8_{\mathrm{N}-\mathrm{C}}$ complex.

Abbreviations: M, marker; MW, molecular weight; F, fraction

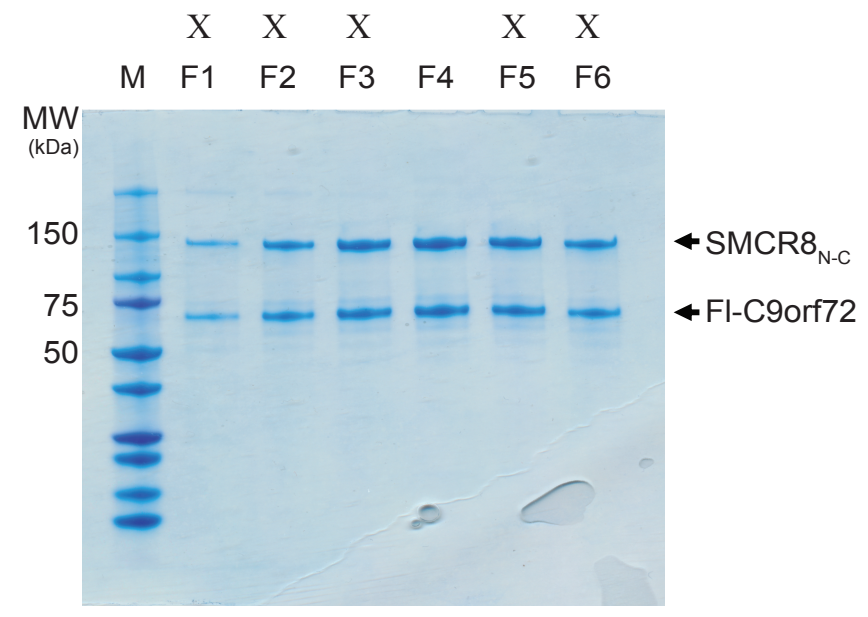




\section{Gel 3}

S1B Fig was generated from this gel.

Uncropped Coomassie stained SDS-PAGE gel analyzing the peak fraction of the proteolyzed full length trimeric C9orf72 complex obtained after size-exclusion chromatography.

The C9orf72 complex was eluted in one peak fraction, which was then analyzed on SDS-PAGE gel, using various protein concentrations. Bands, labelled from 1-10, were subsequently excised and analyzed by mass spectrometry. The identity of each band is:

1. Fl-SMCR8, 2. Fl-C9orf72, 3. Fl-WDR41, 4. Hsp70, 5. Fl-C9orf72 and SMCR8 ${ }_{\mathrm{N}}$, 6. parts of SMCR8 and Fl-WDR41, 7. SMCR8 ${ }_{\mathrm{N}}$ and $\mathrm{SMCR} 8_{\mathrm{C}}, 8 . \mathrm{SMCR} 8_{\mathrm{C}}$, 9. central SMCR8, 10. peptides from all the proteins.

Abbreviations: M, marker; MW, molecular weight

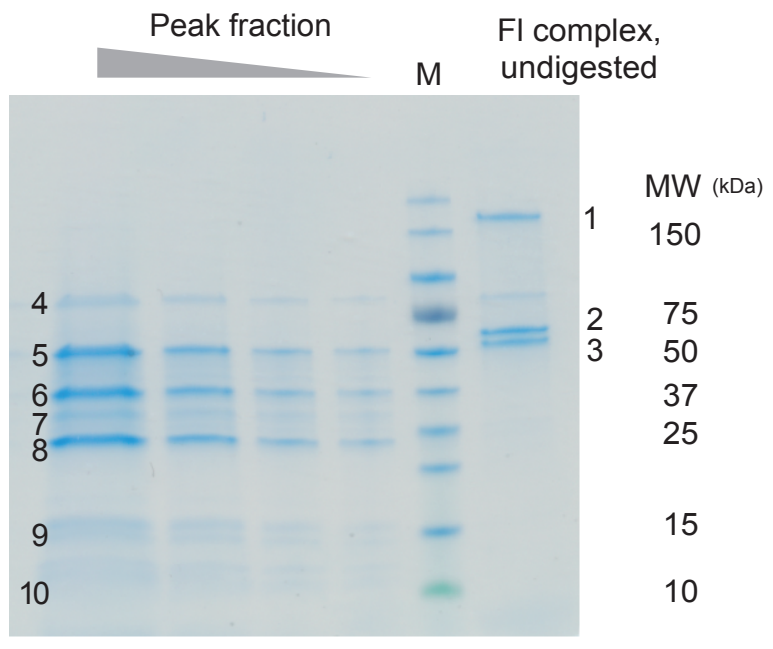




\section{Gel 4}

Fig 3A top panel was generated partly from this gel.

Non-included lanes are marked with an ' $\mathrm{X}$ '.

Uncropped Coomassie stained SDS-PAGE gel analyzing the input of the pulldown assay of purified WDR41 with the binary complex containing GST-C9orf72 and Fl-SMCR8 or SMCR ${ }_{\mathrm{N}}$.

GST alone was used to assess background binding of WDR41 to the tag.

Two different GST-pulldowns (1 and 2) were carried out, which differ in WDR41 that came from two different purifications. For Fig 3A, pulldown 2 was used, which in this gel corresponds to input 2 . Used lanes are numbered equally as in Fig $3 \mathrm{~A}$ top (left part).

Abbreviations: M, marker; MW, molecular weight

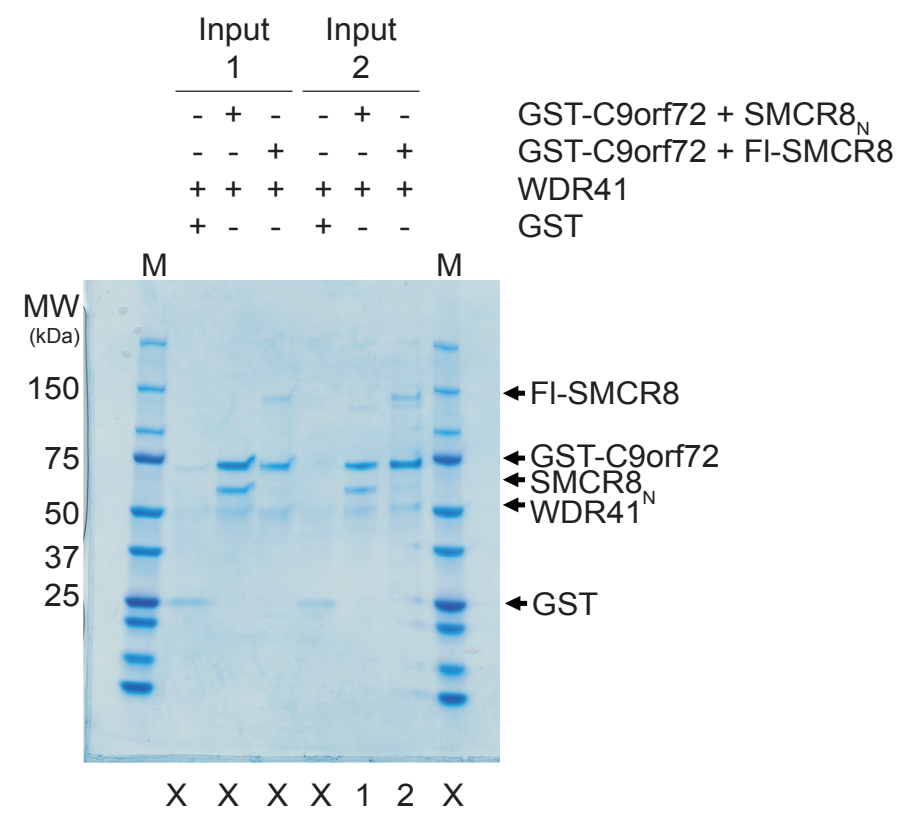




\section{Gel 5}

Fig 3A top panel was generated partly from this gel.

Non-included lanes are marked with an ' $\mathrm{X}$ ' .

Uncropped Coomassie stained SDS-PAGE gel analyzing the pulldown assay of purified WDR41 with the binary complex containing GST-C9orf72 and Fl-SMCR8 or SMCR $8_{\mathrm{N}}$.

GST alone was used to assess background binding of WDR41 to the tag.

Two different GST-pulldowns (1 and 2) were carried out, which differ in WDR41 that came from two different purifications. For Fig 3A, pulldown 2 was used.

Used lanes are numbered equally as in Fig $3 \mathrm{~A}$ top (right part).

Abbreviations: M, marker; MW, molecular weight

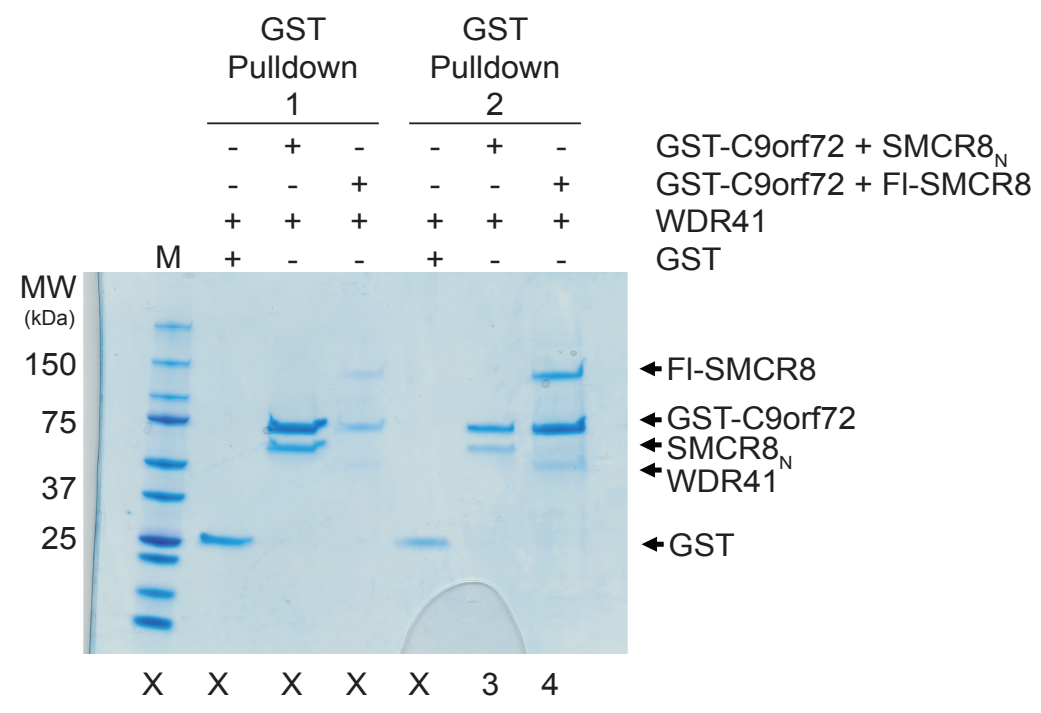




\section{Gel 6}

Fig 3A bottom panel was generated from this gel.

Non-included lanes are marked with an ' $\mathrm{X}$ ' .

Uncropped Coomassie stained SDS-PAGE gel analyzing the pulldown assay of purified WDR41 with the binary complex containing SUMO-C9orf72 and C-terminal MBP-tagged SMCR $8_{\mathrm{N}-\mathrm{C}}$.

MBP alone was used to assess background binding of WDR41 to the tag (corresponds to lane 2 and 4 ).

Used lanes are numbered equally as in Fig 3A bottom.

Abbreviations: M, marker; MW, molecular weight

\begin{tabular}{|c|c|c|c|}
\hline Input & $\begin{array}{c}\text { MBP } \\
\text { Pulldown }\end{array}$ & Supernatant & \\
\hline & + & + & C9orf72 + MBP-SMCR $8_{\mathrm{N}-\mathrm{C}}$ \\
\hline & + & + & MBP \\
\hline & + & $++M$ & WDR41 \\
\hline
\end{tabular}

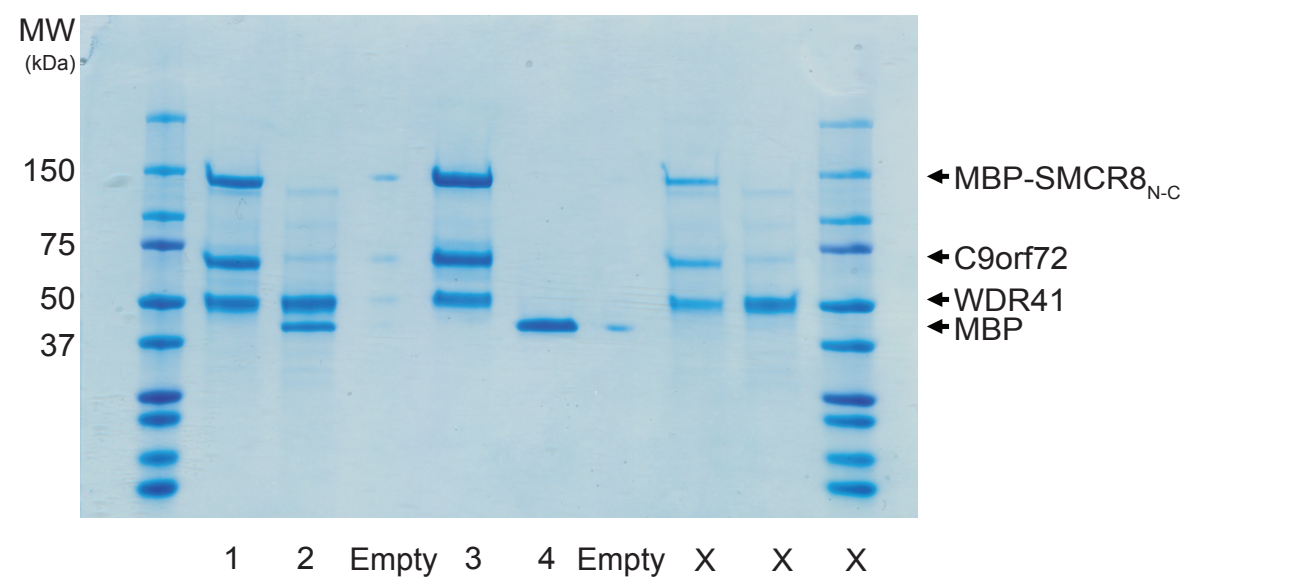

